\title{
Endoscopic Ultrasound-Guided Acquisition of Portal Venous Circulating Tumor Cells as a Potential Diagnostic and Prognostic Tool for Pancreatic Cancer
}

\author{
Yixuan Zhang ${ }^{l, *}$ \\ Haochen $\mathrm{Su}^{2, *}$ \\ Haibo Wang ${ }^{3, *}$ \\ Chenghu $\mathrm{Xu}^{\mathrm{I}}$ \\ Siqi Zhou ${ }^{4}$ \\ Jing Zhao' \\ Shanshan Shen' \\ Guifang Xu' \\ Lei Wang' \\ Xiaoping Zou' \\ Shu Zhang' \\ Ying Lv'
}

'Department of Gastroenterology, Nanjing Drum Tower Hospital, The Affiliated Hospital of Nanjing University Medical School, Nanjing, People's Republic of China; ${ }^{2}$ Department of Gastroenterology, Nanjing Drum Tower Hospital, Clinical College of Nanjing Medical University, Nanjing, People's Republic of China; ${ }^{3}$ Cyttelbio Corporation, Beijing, People's Republic of China; ${ }^{4}$ Department of Gastroenterology, Nanjing Drum Tower Hospital, The Affiliated of Jiangsu University, Nanjing, People's Republic of China

*These authors contributed equally to this work

\begin{abstract}
Background: Circulating tumor cells (CTCs) were a promising liquid biopsy for pancreatic cancer (PC) but circulate in low counts in peripheral blood. We evaluated the diagnostic and prognostic values of portal vein (PoV) CTCs in PC patients.

Methods: PoV was aspirated under EUS guidance from 40 patients with suspected pancreaticobiliary cancers. Epithelial-mesenchymal-transition-related subtypes of CTCs were identified via immunofluorescence using EpCAM and Twist antibodies. The diagnostic and prognostic performance of PoV CTCs was investigated by receiver-operating characteristic (AUC) curve and Kaplan-Meier survival analysis.
\end{abstract}

Results: In total, 40 patients including 31 with PC, 4 with non-pancreatic periampullary cancer and 5 with benign pancreatic diseases (BPD) were enrolled. CTCs were detected more in PoV compared with peripheral blood. PoV CTC numbers in BPD patients were lower than in PC patients. The number of PoV CTCs, especially mesenchymal-CTCs (M-CTCs), was positively correlated with the tumor burden, instead of epithelial-CTCs (E-CTCs). The combination of PoV CTC numbers and CA19-9 demonstrated better diagnostic efficiency (AUC value 0.987) than either alone in differentiating PC with BPD. Moreover, the diagnostic efficacy of PoV CTCs and M-CTCs were obviously better than that of E-CTCs and CA19-9 in distinguishing early and late stage PC. Lastly, high PoV CTC and M-CTC numbers were both associated with shorter overall survival.

Conclusion: Acquisition of the PoV samples in PC patients via EUS-guided procedures has been proved safe and feasible. PoV CTCs, especially M-CTCs, have great potentials in diagnosing and predicting the prognosis of PC, especially in combination with CA19-9.

Keywords: circulating tumor cell, endoscopic ultrasound, portal vein blood, diagnosis, prognosis

\section{Introduction}

Pancreatic cancer (PC) is the most devastating malignant disease with poor prognosis worldwide. ${ }^{1,2}$ Unlike many other cancers, the incidence and mortality of PC are increasing, and PC is predicted to be the second-leading cause of cancer-related deaths worldwide by $2030 .^{3}$ Most patients with symptomatic PC have reached advanced and metastatic stages, making them ineligible for surgical resections. Besides, PC still has a dismal 5-year survival rate below 5\% despite improvement of survival for the patients undergoing surgical resections. ${ }^{4}$ This suggested that
Correspondence: Shu Zhang; Ying Lv Email zhangsgastro@nju.edu.cn; lvying@njglyy.com 
current criteria are not appropriate to select patients who might benefit from surgeries. Currently, carbohydrate antigen 19-9 (CA19-9) has been used widely in clinical practice. However, the sensitivity and specificity of CA19-9 for diagnosing PC, especially for differential diagnosis of PC patients at different stages, are still in doubt. And patients with negative Lewis antigen do not benefit from CA19-9 examination. ${ }^{5}$ Therefore, developing reliable biomarkers that can aid early detection and prognostic judgment is crucial for improving clinical outcome of PC. ${ }^{6}$

Circulating tumor cells (CTCs), recognized as the seeds of distant metastasis, are tumor cells shed from primary tumors and migrate into the circulation causing secondary deposits. ${ }^{7}$ Accumulating evidence have confirmed that CTCs have promising effects in diagnosing PC and predicting metastasis and prognoses of PC patients. ${ }^{8,9}$

The pancreatic vein blood drains first into the liver, causing liver as the most common metastatic site of PC. ${ }^{10,11}$ The filtration effect of the liver made CTCs highly diluted in the peripheral blood, and increased the difficulty of detecting enough CTCs in the peripheral blood samples. ${ }^{12}$ Unlike traditional liquid biopsy, tumorproximal liquid biopsy means obtaining a sample in vessels close to the tumor, especially in the main veins that drain blood from the organ invaded by the cancer, leading to higher capability of capturing and detecting enough CTCs. ${ }^{13}$ The feasibility of the puncture in these vessels has already been successfully tested in patients with colorectal cancer, ${ }^{14}$ liver cancer, ${ }^{15}$ lung cancer $^{16}$ and PC. ${ }^{17}$ However, until now, most researchers obtained portal vein blood from PC patients during pancreaticoduodenectomy rather than minimally invasive access.

Endoscopic ultrasound (EUS) provides high-resolution, precise access to portal vein allowing for minimally invasive access for portal vein blood sampling. ${ }^{18}$ Catenacci et al first reported that portal vein blood can be acquired safely, and an increased number of CTC in the portal vein blood was found compared with peripheral circulation in PC patients. ${ }^{19}$ However, due to the relatively small sample size and heterogeneity of the patients, they did not further investigate the correlation between clinical features and CTCs in the portal vein blood.

The most widely used biomarker to identify CTCs is an epithelial cell marker called epithelial cell adhesion molecule (EpCAM) by far. CellSearch ${ }^{\circledR}$ which is based on the surface antibody-based technique to capture $\mathrm{EpCAM}^{+} \mathrm{CTCs}$ is the first and only FDA-approved CTC detection platform in breast, colorectal, and prostate cancer. ${ }^{20}$ However, the majority of studies have shown that the EpCAM-based identification of CTCs does not fit logically with the theory of epithelial-mesenchymal transition (EMT) in the malignant progression, and may miss these EpCAM-negative CTCs which are believed to be a more aggressive and invasive subtype and are essential for distant metastasis. ${ }^{21-23}$ Therefore, to avoid underestimating the number of CTCs, we decide to capture CTCs with a negative enrichment method, followed by the characterization of the captured CTCs with epithelial biomarker EpCAM and mesenchymal biomarker Twist together with leukocyte common antigen CD45 to exclude remnant white blood cells.

In the present study, we evaluated the safety and feasibility of the trans-hepatic puncture using a $22 \mathrm{G}$ needle to collect portal vein blood in patients with suspected PC referred for EUS-FNA (fine-needle aspiration). Besides, we identified the EpCAM-positive and/or Twist-positive CTCs in the portal vein blood and peripheral blood of PC patients, and analyzed their diagnostic and prognostic values in the clinical practice.

\section{Materials and Methods}

\section{Patients and Clinical Samples}

From July 2019 and July 2020, 40 patients with suspected pancreaticobiliary cancers referred for EUS-FNA were enrolled into this prospective study. All included patients had a negative history of blood transfusion, acquired immunologically mediated disease or any anti-tumor treatment before enrollment. Classification of PC was assessed using the 8th edition of the American Joint Committee on Cancer (AJCC) Classification. Follow-up was made by telephone call every 2 months and terminated on January 2021, and none of the patients lost to follow-up during the study. Patients were followed completely and overall survival (OS) was defined as the time interval between the date of EUS procedure when portal vein blood samples were obtained and the date of death or the end of follow-up.

All patients signed informed consent before the procedure. This study was conducted in compliance with the institutional policy to protect private patient information and was approved by the institutional review board of the Ethics Committee of Nanjing Drum Tower Hospital affiliated to Nanjing University Medical School (approval number: 2018-076-01). Our clinical studies are registered on clinicaltrials.gov (ID: NCT03821909). The study was performed in accordance with the principles of Declaration of Helsinki. 


\section{Portal Vein Sampling}

Under EUS guidance, the left and right portal veins were identified. After verifying flow signal by Doppler, a 22gauge EUS fine needle was subsequently punctured transhepatically into the portal vein (Figure 1) and a 5-mL sample of portal vein blood was aspirated. Puncture site complications were monitored under EUS. Meanwhile, 5-mL peripheral blood was obtained before EUS in parallel.

\section{Isolation and Identification of CTCs}

Blood samples were processed within $4 \mathrm{~h}$ of collection. CTCs were isolated using the Cyttel Detection kit (CS1, CS2, CS3, and CF1 solutions; Cyttel Biosciences INC, Jiangsu, China). Briefly, a 5-mL sample of peripheral or portal vein blood was collected in a BD Vacutainer tube (Becton, Dickinson and Company, Franklin, NJ) and was washed once with CS1. The red blood cells in sample were then lysed using CS2 and separated by centrifugation at $650 \mathrm{~g}$ for $5 \mathrm{~min}$, the resulting cell pellet was re-suspended in CS1. White blood cells bound to the CD45 monoclonal antibody coated magnetic beads were placed on top of CS3 (a special gradient centrifugation liquid) and separated at $300 \mathrm{~g}$ for $5 \mathrm{~min}$. The resulting solution with any CTCs was smeared on a single slide (Thermo Fisher Scientific, Franklin, MA), fixed, and dried for subsequent analysis.

CTC subpopulations were identified using immunofluorescence. Detection of EMT phenotype was performed
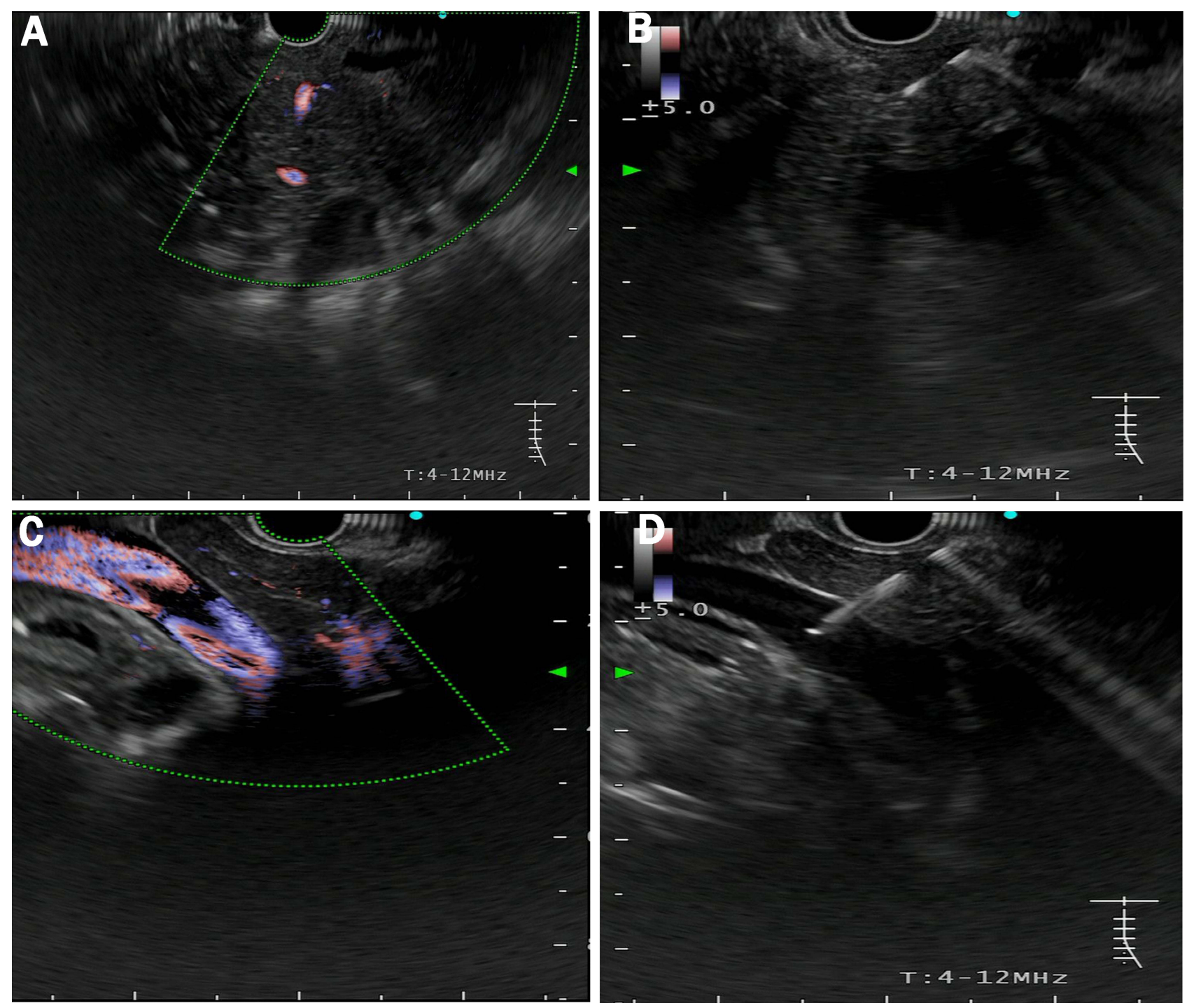

Figure I EUS-guided access of the portal vein. (A) Pancreatic mass was hypoechoic with poorly defined boundaries. (B) EUS-FNA to obtain histological cytology and histological examination. (C) The main portal vein was identified under EUS guidance with Doppler wave verification. (D) Puncture the primary vein with a needle for portal venous blood acquisition. 
using epithelial biomarker EpCAM and mesenchymal biomarkers Twist. The enriched cells were fixed and incubated with the anti-human EpCAM/Twist/CD45 (leukocyte biomarker, leukocyte common antigen) antibody for $2 \mathrm{~h}$. Fluorescein-conjugated secondary antibodies were incubated at $37^{\circ} \mathrm{C}$ for $30 \mathrm{~min}$ and then rinsed with washing buffer. The slides were then mounted using DAPI-containing mounting media and observed using a fluorescence microscope. We defined CTCs as epithelial CTCs showing orange fluorescence with EpCAM expression, while mesenchymal CTCs exhibited green fluorescence with Twist expression. A third hybrid population of CTCs expressing both epithelial- and mesenchymalspecific markers was also observed (Figure 2).

\section{Cell Lines}

The cells were maintained at $37^{\circ} \mathrm{C}$ with $5 \% \mathrm{CO} 2$. Human pancreatic cell lines Panc-1, Miapaca-2, BxPC3 and Sw1990 were obtained from the Cell Bank of Chinese Academy of Sciences (Shanghai, China). All cell lines were authenticated by Short Tandem Repeats profiling and tested for mycoplasma contamination.

\section{Statistical Analysis}

All statistical analyses were performed using SPSS 25.0 (IBM Corp., Armonk, NY, USA) and Prism 8.0 (GraphPad, La Jolla, CA, USA), and a two-tailed $\mathrm{P}$ value $\leq 0.05$ was considered significant. Descriptive statistics were presented as mean values with ranges for continuous variables and as percentages for categorical variables. Differences were compared through the Student's $t$-test or Mann-Whitney $U$-test, and to compare the levels between multi-group analysis, the Kruskal-Wallis test was used. A receiver operating characteristic (ROC) curve and the area under the curve (AUC) were used to describe the diagnostic performance. Differences in OS were plotted using Kaplan-Meier survival plots and tested using the Log rank test. Hazard ratios (HR) and 95\% CI (confidence intervals) were assessed by Cox regression analysis.

\section{Result \\ Patient Characteristics and Protocol Feasibility}

A total of 40 patients with suspected pancreaticobiliary cancers who underwent EUS-FNA were prospectively enrolled in

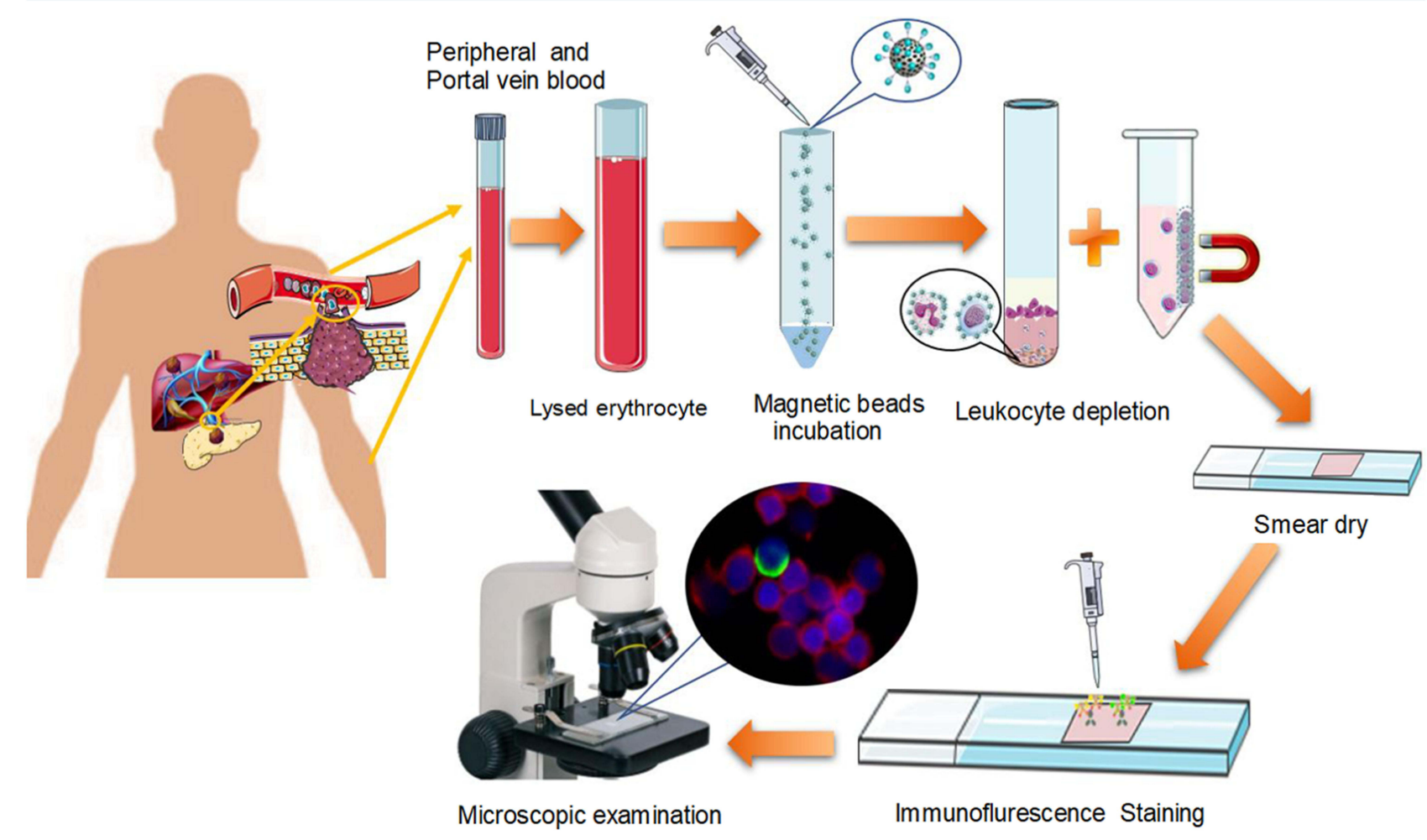

Figure 2 Flowchart of the protocol for CTC detection in our study. The peripheral and portal vein blood were collected from each patient. Red blood Cells (RBCs) in the samples were lysed, and residual cell pellets were resuspended. Samples were labeled with anti-leukocyte-specific antibodies (CD45) monoclonal antibody coated magnetic beads for $20 \mathrm{~min}$, followed by separation of the beads using a gradient centrifugation liquid and magnetic stand. The resulting solution with any CTCs was smeared on a single slide, fixed, and dried for CTC EMT phenotype detection by immunofluorescence. 
this study (Figure 3). The mean age of the patients at the time of diagnosis was 63 years (range, 33-76 years), and they were predominantly females $(n=24,60 \%) .31$ patients were confirmed to have pancreatic cancer (PC), 4 patients with nonpancreatic periampullary cancer (non-PC), including 2 with ampullary carcinoma (AMPC) and 2 with distal bile duct cancer (DBDC), and another 5 patients with benign pancreatic diseases (BPD), including 2 patients with intraductal papillary mucinous neoplasm (IPMN), 1 patient with mucinous cystic neoplasm (MCN), 1 patient with serous cystadenoma ( $\mathrm{SCN}$ ) and 1 patient with neuroendocrine neoplasm (NEN). Of all the PC patients, 9 had stage I-II disease and 22 had stage III-IV disease. Notably, 8 (25.81\%) of the patients had distant metastasis. All the patient characteristics are summarized in Table 1.

In our practice, the FNA entry site is monitored under direct EUS color Doppler visualization for complications for a minimum of $5 \mathrm{~min}$ in the endoscopy suite. Patients were observed in the recovery area for $45 \mathrm{~min}$ after the procedure. Telephone calls were made routinely 24 hours and 7 days after the procedure to assess for post-procedure recovery. None of immediate or delayed complications from the procedure of portal vein blood acquisition, including bleeding or thrombotic events, were observed. Collectively, our results demonstrated the safety and feasibility of the trans-hepatic puncture using $22 \mathrm{G}$ needle to collect portal vein blood in clinic.

\section{Characterization of CTCs in Pancreatic Cancer}

The specificity of the antibodies for epithelial (EpCAM) and mesenchymal (Twist) markers were initially validated in various PC cell lines. Panc-1 and Miapaca-2 displayed high expression of mesenchymal markers, while less invasive cell lines, such as SW1990 and Bxpc3 demonstrated the opposite expression profile (Figure 4A).

Subsequently, we succeeded in detecting CTCs in the portal vein of patients with PC. CTCs were divided into the following three subtypes based on the expression profiles of epithelial and mesenchymal markers as M-CTCs (mesenchymal subtype, EpCAM $/$ Twist $^{+} / \mathrm{CD}^{-} 5^{-} / \mathrm{DAPI}^{+}$), H-CTCs (hybrid subtype, $\mathrm{EpCAM}^{+} /$Twist $^{+} / \mathrm{CD}^{-} 5^{-} / \mathrm{DAPI}^{+}$) or E-CTCs (epithelial subtype, $\mathrm{EpCAM}^{+} /$Twist ${ }^{-} / \mathrm{CD}^{-} 5^{-} / \mathrm{DAPI}^{+}$) (Figure 4B).

Taken together, these data indicated the flexibility of our platform to identify EMT characterization of CTCs in patients with PC.

\section{Validation of CTC Numbers in Portal Vein Blood as a Potential Biomarker for Pancreatic Cancer Diagnosis}

Among all 31 patients with PC, CTCs were detected in 30 patients (97\%) with PC in portal vein blood (PoV), while 27 patients $(87 \%)$ had detectable CTCs in peripheral blood. The

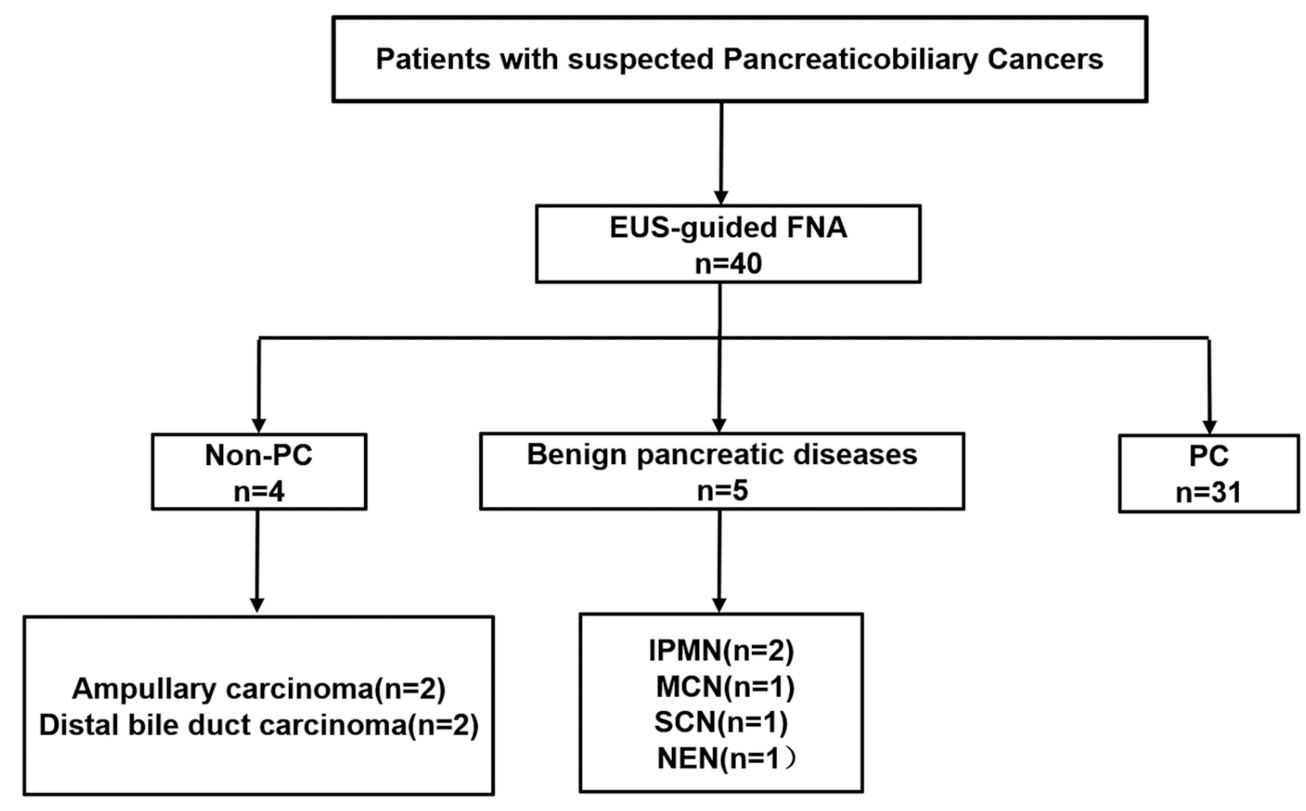

Figure 3 Flowchart of the enrolled patients.

Abbreviations: EUS, endoscopic ultrasound; FNA, fine-needle aspiration; Non-PC, non-pancreatic periampullary cancer; PC, pancreatic cancer; IPMN, intraductal papillary mucinous neoplasm; MCN, mucinous cystic neoplasm; SCN, serous cystadenoma; NEN, neuroendocrine neoplasm. 
Table I Patient Characteristics

\begin{tabular}{|c|c|}
\hline \multicolumn{2}{|l|}{ Characteristics } \\
\hline$P C(n=3 I)$ & \\
\hline Age, mean (range, years) & $63(43-76)$ \\
\hline Sex (\%) & \\
\hline Male & $12(38.70 \%)$ \\
\hline Female & $19(61.30 \%)$ \\
\hline Tumor size, mean (range, mm) & $31,75(20.00-42.80)$ \\
\hline AJCC stage (\%) & \\
\hline 1 & 7 (22.58\%) \\
\hline II & $2(6.45 \%)$ \\
\hline III & $14(45.16 \%)$ \\
\hline IV & $8(25.81 \%)$ \\
\hline Tumor location (\%) & \\
\hline Head or neck & 18 (58.06\%) \\
\hline Body or tail & 13 (41.94\%) \\
\hline Lymph nodes status (\%) & \\
\hline Positive & $14(45.16 \%)$ \\
\hline Negative & 13 (41.94\%) \\
\hline $\mathrm{Nx}$ & $4(12.90 \%)$ \\
\hline Vascular infiltration (\%) & \\
\hline No & $16(51.61 \%)$ \\
\hline Yes & 15 (48.39\%) \\
\hline Metastasis (\%) & \\
\hline No & $23(74.19 \%)$ \\
\hline Yes & $8(25.80 \%)$ \\
\hline Non-PC $(n=4)$ & \\
\hline Age, mean (range, years) & $64.75(62-67)$ \\
\hline Sex (\%) & \\
\hline Male & $2(50.00 \%)$ \\
\hline Female & $2(50.00 \%)$ \\
\hline Tumor size, mean (range, mm) & $29.90(20.80-37.60)$ \\
\hline Histopathologic type & \\
\hline Ampullary carcinoma & $2(50.00 \%)$ \\
\hline Distal bile duct carcinoma & $2(50.00 \%)$ \\
\hline Benign diseases $(n=5)$ & \\
\hline Age, mean (range, years) & $58(33-70)$ \\
\hline Sex (\%) & \\
\hline Male & $2(40 \%)$ \\
\hline Female & $3(60 \%)$ \\
\hline Tumor size, mean (range, mm) & $34.18(11.70-60.20)$ \\
\hline Histopathologic type & \\
\hline IPMN & $2(40 \%)$ \\
\hline $\mathrm{MCN}$ & I (20\%) \\
\hline $\mathrm{SCN}$ & I (20\%) \\
\hline NEN & I (20\%) \\
\hline
\end{tabular}

Abbreviations: PC, pancreatic cancer; Non-PC, non-pancreatic periampullary cancer; IPMN, intraductal papillary mucinous neoplasm; $\mathrm{MCN}$, mucinous cystic neoplasm; SCN, serous cystadenoma; NEN, neuroendocrine neoplasm.
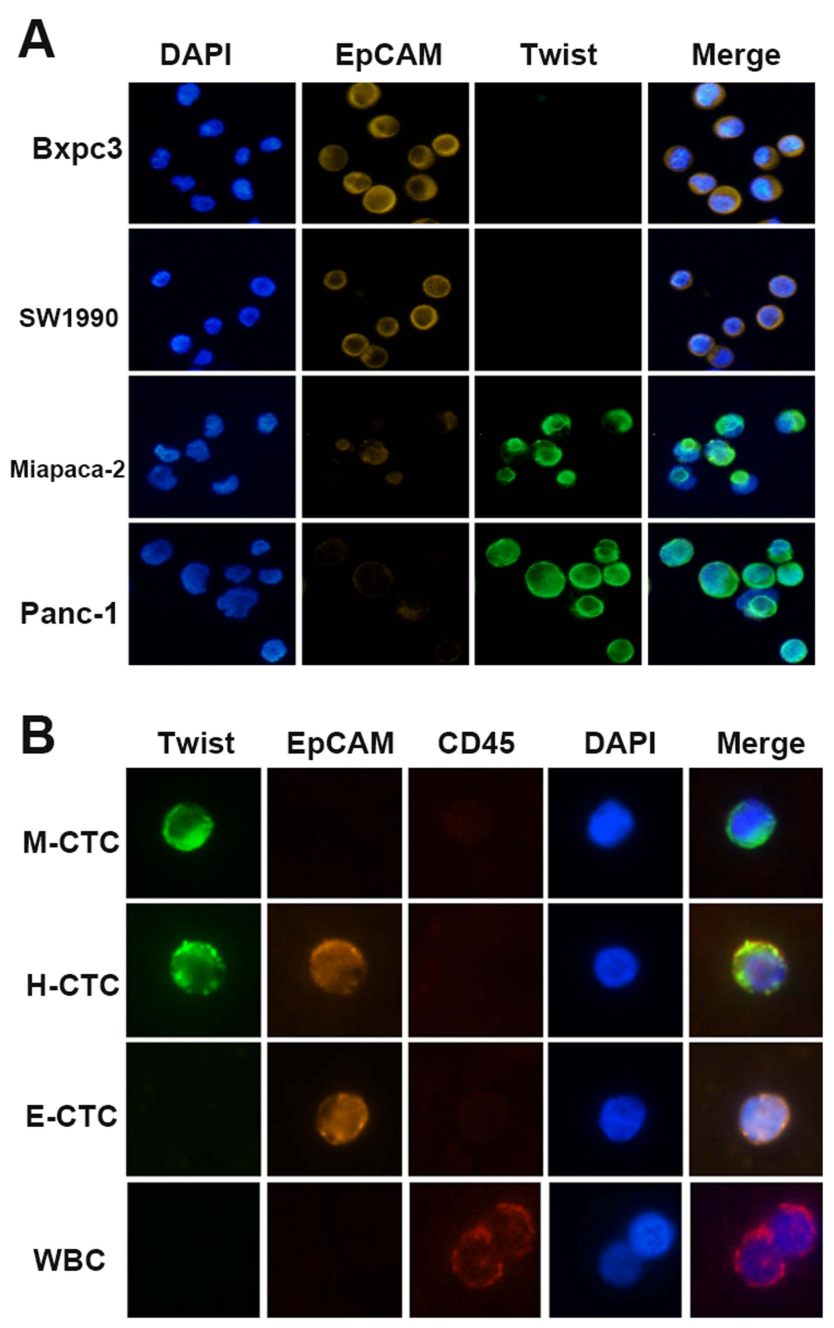

Figure 4 Verification and detection of EMT phenotype in PC cell lines and portal vein CTCs from PC patients. (A) EMT phenotype of this platform was verified in representative "epithelial" (Bxpc3, sw 1990) and "mesenchymal" (Miapaca-2, Panc-I) PC cell lines. The cells were analyzed using a 200X× objective. (B) Representative images of CTCs in portal vein blood from a patient with PC. Leukocytes were stained for CD45 (red fluorescence). CTCs were stained for epithelial markers (EpCAM, Orange fluorescence) and mesenchymal markers (Twist, green fluorescence). The cells were analyzed using a $400 x$ objective.

number of CTCs in PoV was higher than in peripheral blood (PV) in each patient indicated by paired analysis (median, 10 vs 6 cell $/ 5 \mathrm{~mL}$ blood) (Figure $5 \mathrm{~A}, \mathrm{p}<0.0001$ ). To further validate the specificity of CTCs in $\mathrm{PoV}$ as a biomarker for PC, we examined the number of CTCs in PC, BPD and nonPC, respectively. According to the above definition, the number of CTCs was 0-36 CTCs $/ 5 \mathrm{~mL}$ (median, $10 \mathrm{CTCs} /$ $5 \mathrm{~mL}$ ) in PC patients, $0-4 \mathrm{CTCs} / 5 \mathrm{~mL}$ (median, $2 \mathrm{CTCs} / 5 \mathrm{~mL}$ ) in $\mathrm{BPD}$ and $8-14 \mathrm{CTCs} / 5 \mathrm{~mL}$ (median, $11 \mathrm{CTCs} / 5 \mathrm{~mL}$ ) in non-PC. The total CTC numbers in patients with BPD were significantly lower than those in patients with $\mathrm{PC}(\mathrm{p}=0.004)$; however, no obvious difference of the CTC numbers in PoV was observed between PC and non-PC groups (Figure 5B). 

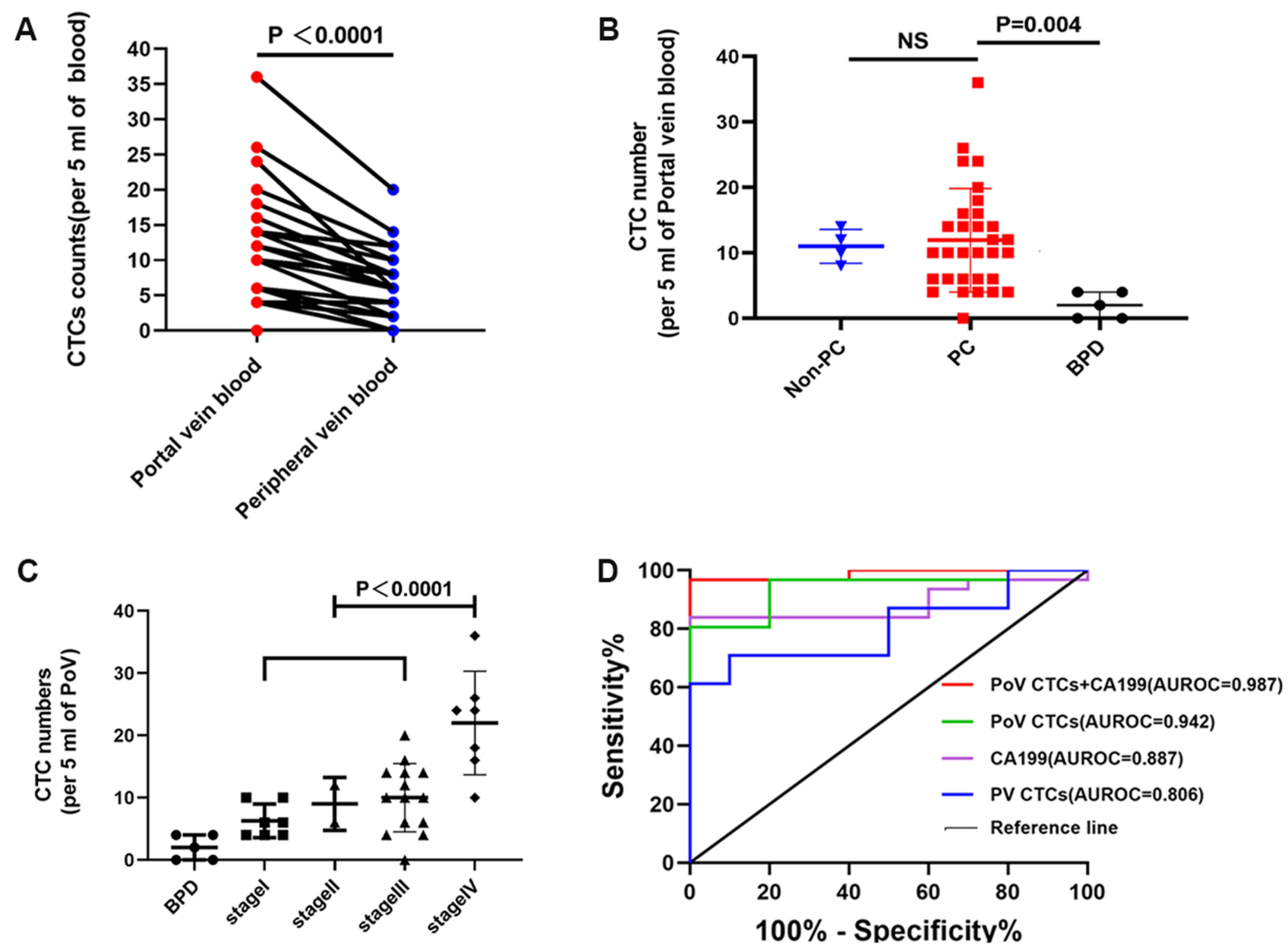

Figure 5 Circulating tumor cells (CTCs) in portal vein blood (PoV) constitute a potential biomarker for PC. (A) CTC numbers in PoV were significantly higher than in peripheral blood (median, 10 vs 6 cell $/ 5 \mathrm{~mL}$ of blood, $\mathrm{p}<0.000$ ); (B) Comparison of PoV CTC numbers in patients with PC, non-PC and BPD. PC, pancreatic cancer; nonPC, non-pancreatic periampullary cancer; BPD, benign pancreatic diseases. (C) CTC numbers among patients with BPD, and PC patients at different stages. (D) The diagnostic performance of PoV CTC numbers, PV CTC numbers, CA 19-9, and combined PoV CTC numbers with CAI9-9 in differentiating PC and BPD was shown by ROC curves.

Next, we investigated the CTC numbers of PoV in PC patients at different stages. As shown in Figure 5C, CTCs were rare in $\mathrm{BPD}$, and it was obvious that the $\mathrm{CTC}$ numbers in PoV at the latest stage of PC were significantly higher than stage I-III groups $(p<0.0001)$. Importantly, in stage I-III PC patients, the increasing trend in the number of CTCs was stage-dependently (Figure 5C). We further constructed receiver operating characteristic (ROC) curve to assess the utility of the CTC number in PoV (combined with or without serum CA19-9 levels) as a diagnostic tool for differentiating PC and BPD.

For all the patients, at the optimized cut-off of $6 \mathrm{PoV}$ CTCs/5mL, PoV CTCs were able to distinguish patients with BPD from those with PC, with a sensitivity of $80.6 \%$, specificity of $100.0 \%$, and AUROC of 0.942 (95\% CI 0.864 $-1.000)$. Similarly, CA199, at the optimum cut-off of
47.43U $/ \mathrm{mL}$, distinguished patients with BPD, with a sensitivity $83.9 \%$, specificity of $100.0 \%$, and AUROC of 0.887 (95\% CI 0.777-0.997) (Figure 5D). Moreover, the combination of PoV CTCs and CA19-9, at the optimum cutoff of 0.99 , distinguished patients with BPD from those with PC, with a sensitivity of $84.0 \%$ and specificity of $100.0 \%$, and AUROC of 0.987 (95\% CI 0.956-1.000). For comparison, at the optimized cut-off of $5 \mathrm{CTCs} / 5 \mathrm{~mL}$, PV CTCs were able to distinguish patients with BPD, with a sensitivity of $61.3 \%$, specificity of $100.0 \%$, and AUROC of 0.806 (95\% CI 0.654-0.958). Notably, we found that the diagnostic power of PV CTCs was relatively poor compared with PoV CTCs and CA19-9. Therefore, we suggested that the PoV CTC numbers had promising potentials in PC diagnosis, and the combination of PoV CTCs and serum CA19-9 could further improve the diagnostic value. 


\section{Numbers of CTCs, Especially} Mesenchymal CTCs in the Portal Vein Blood, Correlate with the Tumor Burden In our 31 patients with $\mathrm{PC}$, we noticed that the mesenchymal CTCs (M-CTC) were more frequently detected than epithelial CTCs (E-CTC) in PoV (median, 8 vs 3 cell $/ 5 \mathrm{~mL}$ blood) (Figure 6A, p<0.0001). Furthermore, we analyzed the correlation of the numbers of PoV CTC and M-CTC with the clinical characteristics of all PC patients, and the results are shown in Table 2. In the group comparisons (Figure 6B), the lymph node invasion positive group showed higher levels of PoV CTCs than the negative group ( $p=0.005$ ). Besides, the total PoV CTC numbers in the metastatic group $(23$ cells $/ 5 \mathrm{~mL})$ were obviously enhanced compared with non-metastatic group (8 cells/ $5 \mathrm{~mL}, \mathrm{p}<0.0001)$. What's more, the PoV CTC numbers were markedly increased in late-stage patients (TNM stage III-IV) than in early-stage patients (TNM stage I-II) $(p=0.010)$. In parallel, the same trend of differences existed among all the comparisons for M-CTCs in PoV
(Figure 6C). However, no obvious differences of E-CTC numbers in PoV and total CTC numbers in PV blood were observed between patients with different lymph node status or TNM stages (Figure 6D and E). Moreover, we constructed the ROC curve analysis to distinguish patients with stage I-II from those at the stage of III-IV (Figure 6F and G). The AUC values of CTCs, M-CTCs, E-CTCs numbers in PoV and serum CA19-9 were 0.790 (95\% CI 0.634-0.946), 0.788 (95\% CI 0.632-0.944), 0.652 (95\% CI 0.4637-0.839), and 0.750 (95\% CI 0.569-0.931), respectively. Interestingly, the diagnostic efficacy of PoV CTCs and M-CTCs were obviously better than that of E-CTCs and serum CA19-9. More importantly, the AUC values of combined CTCs or M-CTCs with serum CA19-9 were 0.833 (95\% CI $0.693-0.974)$ and 0.828 (95\% CI 0.682-0.975), respectively, better than the diagnostic efficacy of CTCs or M-CTCs alone (Figure 6F and G). What's more, we also noticed that the diagnostic power of PV CTCs was relatively poor with an AUC value of 0.702 (95\% CI 0.509-0.895) compared with PoV CTCs and CA19-9. Taken together, these data demonstrated that the
A

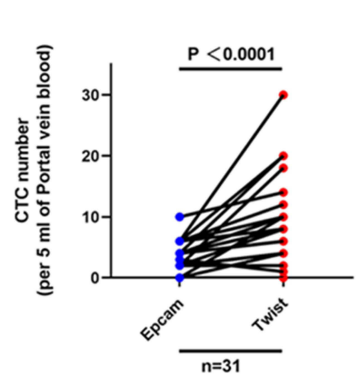

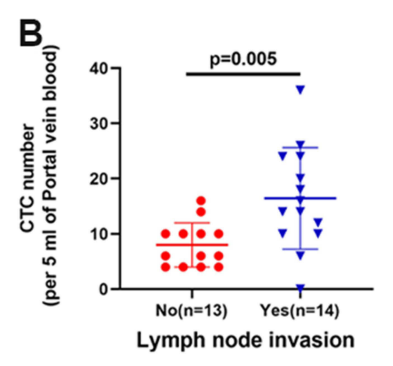

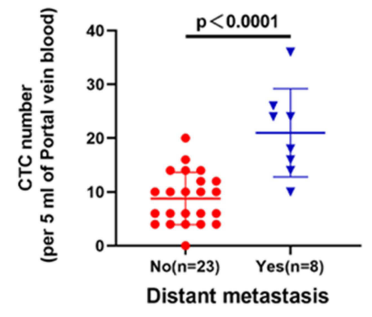

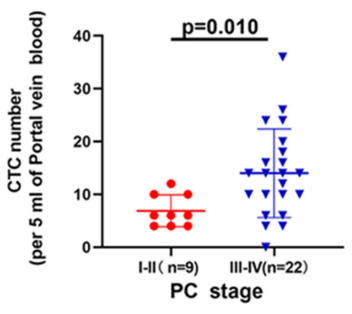

C
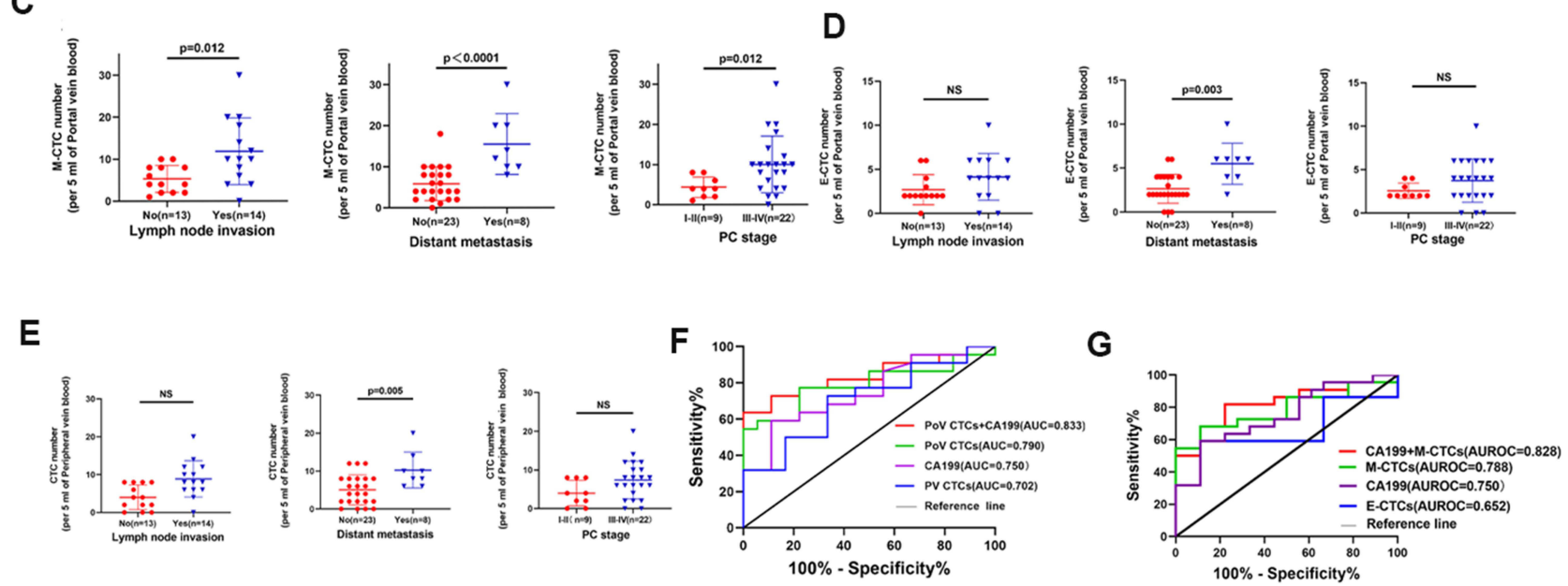

Figure 6 Correlation of the number of CTCs, M-CTCs and E-CTCs in PoV and the number of CTCs in PV with the clinical features of PC patients. (A) The comparison of PoV CTC numbers detected using Twist and EpCAM antibodies. (B-E) Correlation of the numbers of CTCs (B) M-CTCs (C) E-CTCs (D) in portal vein blood and the numbers of CTCs in peripheral vein blood (E) with lymph node invasion, distant metastasis and PC stages. (F and $\mathbf{G})$ ROC curves of CTCs, M-CTCs, E-CTCs in portal vein blood, CTCs in peripheral blood and serum CAI9-9 to distinguish PC patients between stage I-II and III-IV. 
Table 2 The Correlation Between Clinicopathological Characteristics and Numbers of CTCs or M-CTCs in Portal Vein Blood

\begin{tabular}{|c|c|c|c|c|c|}
\hline \multirow[t]{2}{*}{ Characteristics } & \multirow[t]{2}{*}{ No. } & \multicolumn{2}{|c|}{ СTC } & \multicolumn{2}{|c|}{ М-СТC } \\
\hline & & Count (per $5 \mathrm{~mL}$ ) & P value & Count (per $5 \mathrm{~mL}$ ) & $P$ value \\
\hline All patients & 31 & & & & \\
\hline Sex & & & 0.509 & & 0.435 \\
\hline Male & 12 & $24(22-30)$ & & $18(16-24)$ & \\
\hline Female & 19 & $4(4-7)$ & & $8(4-10)$ & \\
\hline Age (years) & & & 0.892 & & 0.953 \\
\hline$>65$ & 16 & $10(7-23)$ & & $8(4-10)$ & \\
\hline$\leq 65$ & 15 & $24(15-25)$ & & $8(4-10)$ & \\
\hline Tumor location & & & 0.859 & & 0.953 \\
\hline Head or neck & 18 & $4(4-7)$ & & $8(3.5-10)$ & \\
\hline Body or tail & 13 & $24(15-30)$ & & $6(4-12)$ & \\
\hline Tumor size $(\mathrm{mm})$ & & & 0.975 & & 0.875 \\
\hline$>40$ & 3 & $10(6-17)$ & & $6(4-10)$ & \\
\hline$\leq 40$ & 28 & $24(15-25)$ & & $8(4-10)$ & \\
\hline AJCC stage & & & $0.010 *$ & & $0.012 *$ \\
\hline I-II & 9 & $6(5-9)$ & & $4(2-6)$ & \\
\hline III-IV & 22 & $24(17-30)$ & & $10(4-12)$ & \\
\hline Lymph nodes status & & & $0.005 *$ & & $0.012 *$ \\
\hline Positive & 14 & $24(17-30)$ & & $10(6-18)$ & \\
\hline Negative & 13 & $4(4-7)$ & & $4(2-8)$ & \\
\hline Vascular infiltration & & & $0.006 *$ & & $0.008 *$ \\
\hline No & 14 & $6(4-12)$ & & $4(2.5-8)$ & \\
\hline Yes & 17 & $14(10-24)$ & & $10(7-16)$ & \\
\hline Metastasis & & & $<0.0001$ & & $<0.0001$ \\
\hline No & 23 & $4(4-7)$ & & $4(3-8)$ & \\
\hline Yes & 8 & $26(25-3 I)$ & & $13(10-20)$ & \\
\hline CAI9-9 level (U/mL) & & & 0.908 & & 0.945 \\
\hline$>37$ & 23 & II (7-23) & & $8(4-10)$ & \\
\hline$\leq 37$ & 8 & $6(4.5-14.5)$ & & $6(4-14)$ & \\
\hline
\end{tabular}

Note: ${ }^{*} \mathrm{p}<0.05$.

number of PoV CTCs, especially M-CTCs, was positively correlated with the tumor burden, and had greater potentials in distinguishing early and late PC patients compared with PV CTCs.

\section{Numbers of CTCs, Especially Mesenchymal CTCs in Portal Vein Blood, are Prognostic Markers in PC Patients}

To evaluate the potential of CTCs and M-CTCs as predictors for cancer prognosis, we investigated their relevance with the overall survival (OS) in the $31 \mathrm{PC}$ patients. Since there is currently no uniform criterion to define high/low CTC numbers, we used the Youden Index (= sensitivity + specificity-1) to determine the threshold for differentiating high vs low CTCs in this work. We found that both total CTC numbers and M-CTC numbers in PoV had good performance, with a cut-off value of $10 /$ $5 \mathrm{~mL}$ (sensitivity $=70.3 \%$, specificity $=100.0 \%$ ) and $8 / 5 \mathrm{~mL}$ (sensitivity $=68.2 \%, \quad$ specificity $=77.8 \%$ ), respectively. Kaplan-Meier survival analysis showed that patients with high CTCs and high M-CTCs in PoV had significantly decreased OS compared with patients with low CTCs $(p=0.004$, Figure 7A) and M-CTCs $(p=0.007$, Figure 7B). 
A
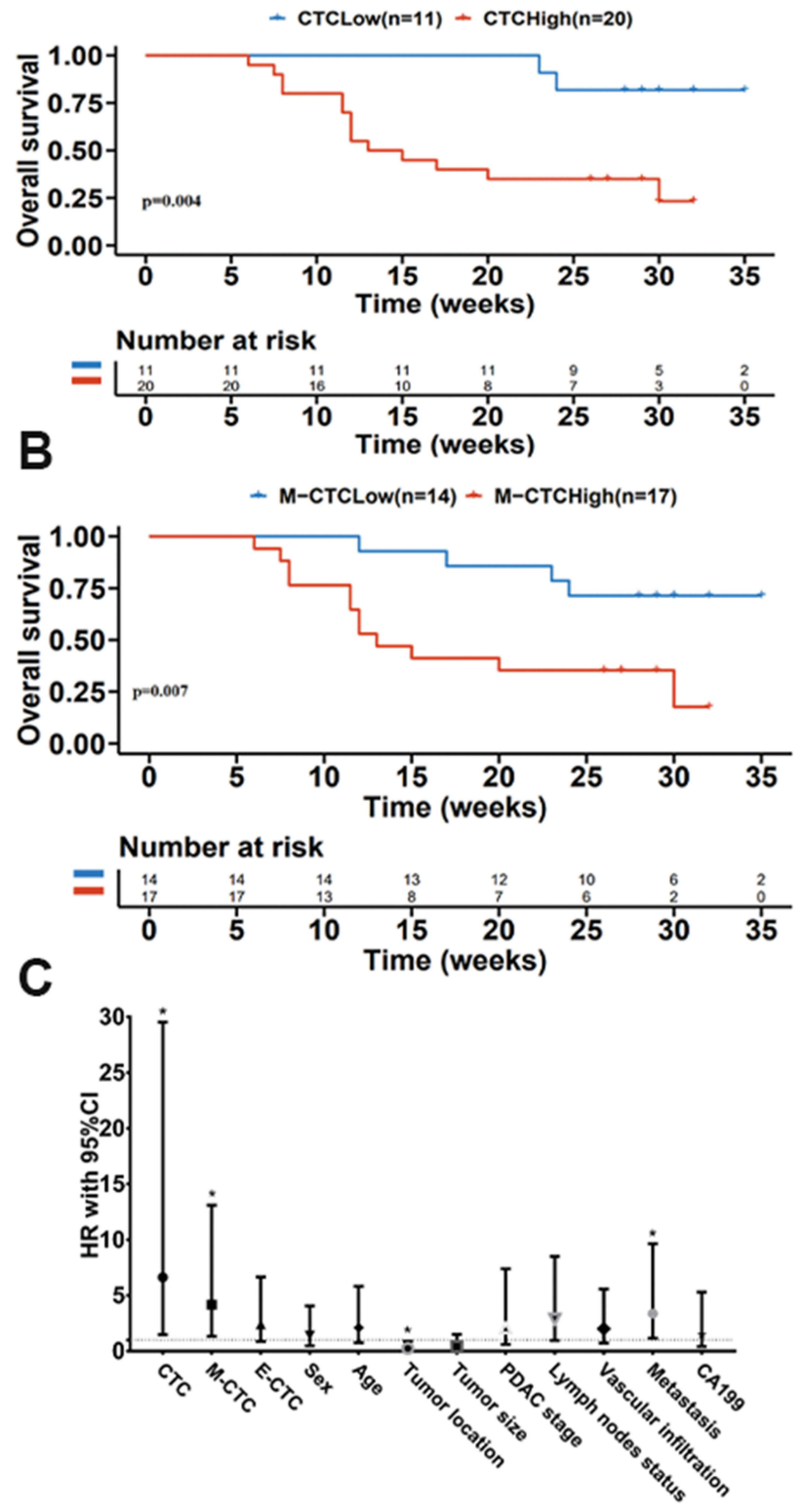

Figure 7 Overall survival (OS) in patients with different levels of CTCs and M-CTCs in PoV. (A) Kaplan-Meier survival analysis showed that patients with lower CTC (CTCs $<10 / 5 \mathrm{~mL})$ had better OS compared with higher CTC (CTCs $\geq 10 / 5 \mathrm{~mL})(p=0.004)$. (B) OS in patients with lower M-CTC $(M-C T C s<8 / 5 \mathrm{~mL})$ was superior to those with higher M-CTC (M-CTCs $\geq 8 / 5 \mathrm{~mL})(p=0.007)$. (C) The hazard ratio $(\mathrm{HR})$ with $95 \% \mathrm{Cl}$ of the CTCs and M-CTCs parameters and clinicopathological characteristics for OS in $\mathrm{PC}$ patients based on COX regression analysis.

The COX regression analysis revealed that patients with CTCs and M-CTCs numbers over the cutoff value had inferior OS (Figure 7C and Table 3), including CTC numbers $(\mathrm{HR}=6.638, \quad \mathrm{p}=0.013), \quad \mathrm{M}-\mathrm{CTC}$ numbers $\quad(\mathrm{HR}=4.172$, $\mathrm{p}=0.014)$, tumor location $(\mathrm{HR}=0.253, \mathrm{p}=0.033)$, and metastasis $(H R=3.377, p=0.023)$. These data supported the number of PoV CTCs and M-CTCs as prognostic markers in PC patients.

\section{Discussion}

In the present study, we reported the safety and feasibility of EUS-guided acquisition of PoV for measurement of CTCs. None of immediate or delayed adverse events from the procedure of portal vein sampling were observed. Although sample size of the pilot study is relatively small, we suggest that the number of $\mathrm{PoV}$ CTCs, especially M-CTCs, might be a valuable tool for early diagnosis, risk stratification and prognosis prediction for PC patients.

To our knowledge, we are the second after Catenacci et $\mathrm{al}^{19}$ to demonstrate that the minimally invasive EUSguided acquisition of PoV for CTC enumeration and isolation is feasible and safe in PC, and PoV CTC can serve as a biomarker for early diagnosis and prognosis for PC. Due to the challenging nature of the procedure, there are no other studies reporting EUS acquired PoV sampling. ${ }^{24}$

PC remains one of the deadliest cancers with poor prognosis due to lack of specific symptoms and early biomarkers for this highly aggressive disease. ${ }^{25}$ In parallel with the search for new strategies to treatment, the most significant challenge is to discover novel biomarkers to ensure early detection, predict prognosis, and help risk stratification. ${ }^{26}$ CTCs, as a non-invasive, real-time blood biomarker, have received concerns in various types of malignancies, including metastatic breast, ${ }^{27}$ prostate, $^{28}$ colon $^{29}$ and pancreatic cancers. ${ }^{30}$ However, application of CTCs in PC has mainly been performed on peripheral blood, and it is noteworthy that, even for advanced patients, CTCs are likely to be more abundant in PoV before being filtered by the liver. ${ }^{31}$ Thus, to increase the chances of CTC detection, blood was sampled directly from the portal vein. Until now, most researchers have obtained PoV from PC patients during surgeries. ${ }^{32,33}$ However, some limitations of intraoperative PoV collection should be noted. First, manipulation of pancreas during resection may release CTCs, leading to the inaccuracy of CTC detection. ${ }^{34}$ Second, only about $15 \%$ of PC patients are eligible for surgical resection. ${ }^{26}$ EUS provides high-resolution, precise access to portal vein allowing for minimally invasive access for PoV sampling, and all patients with various pathological stages can be enrolled. Therefore, obtaining CTCs from PoV via EUS guidance appears to merit significant considerations given the limitations of intraoperative PoV sampling.

Pairwise analysis of CTC counts between PoV and peripheral blood samples showed that the numbers of 
Table 3 Cox Regression Analysis of OS in Pancreatic Cancer

\begin{tabular}{|c|c|c|c|}
\hline Variables & Hazard Ratio & $95 \% \mathrm{Cl}$ & $P$ value \\
\hline $\begin{array}{l}\text { CTCs in PoV }(5 \mathrm{~mL}) \\
\quad \geq 10 \\
\quad<10\end{array}$ & 6.638 & I.49I-29.545 & $0.013^{*}$ \\
\hline $\begin{array}{l}\text { M-CTCs in PoV }(5 \mathrm{~mL}) \\
\geq 8 \\
<8\end{array}$ & 4.172 & $1.328-13.109$ & $0.014^{*}$ \\
\hline $\begin{array}{l}\text { Sex } \\
\qquad \text { Male } \\
\text { Female }\end{array}$ & 1.406 & $0.487-4.057$ & 0.528 \\
\hline $\begin{array}{l}\text { Age (years) } \\
\quad>65 \\
\quad \leq 65\end{array}$ & 2.108 & $0.764-5.814$ & 0.150 \\
\hline $\begin{array}{l}\text { Tumor location } \\
\text { Head or neck } \\
\text { Body or tail }\end{array}$ & 0.253 & $0.072-0.896$ & $0.033^{*}$ \\
\hline $\begin{array}{l}\text { Tumor size }(\mathrm{mm}) \\
\qquad 40 \\
\leq 40\end{array}$ & 0.422 & $0.118-1.504$ & 0.183 \\
\hline $\begin{array}{l}\text { AJCC stage } \\
\text { I-II } \\
\text { III-IV }\end{array}$ & 2.106 & $0.598-7.409$ & 0.246 \\
\hline $\begin{array}{l}\text { Lymph nodes status } \\
\text { Positive } \\
\text { Negative }\end{array}$ & 2.87 & $0.968-8.510$ & 0.057 \\
\hline $\begin{array}{l}\text { Vascular infiltration } \\
\text { No } \\
\text { Yes }\end{array}$ & 2.019 & $0.732-5.57 \mid$ & 0.175 \\
\hline $\begin{array}{l}\text { Metastasis } \\
\text { No } \\
\text { Yes }\end{array}$ & 3.377 & I. $180-9.66 \mid$ & $0.023^{*}$ \\
\hline $\begin{array}{l}\text { CAI9-9 level }(\mathrm{U} / \mathrm{mL}) \\
>37 \\
\leq 37\end{array}$ & $1.50 \mathrm{I}$ & $0.426-5.290$ & 0.528 \\
\hline
\end{tabular}

Note: $* \mathrm{p}<0.05$

CTCs were significantly higher in PoV, consistent with the results of previous studies. ${ }^{19,33,35}$ The number of CTCs in patients with PC was obviously increased compared with those with BPD, and no obvious difference of the CTC numbers in PoV was observed between $\mathrm{PC}$ and non-PC patients. Encouragingly, the combination of PoV CTC numbers and serum CA19-9 improved the specificity and sensitivity of PC diagnosis compared with either CTC or CA19-9 alone.
The majority of studies have identified CTCs by detecting the expression of EpCAM. And the CellSearch system is the only US FDA-approved platform for CTC enumeration, which defines a CTC according to the positive EpCAM expression and negative CD45 expression. ${ }^{20}$ However, EpCAM-based identification of CTCs has limited the positive rate of CTCs and gone against the theory of EMT process. ${ }^{21}$ Besides, it has already been shown that low detection rates were reported by CellSearch system in 
patients with locally advanced PC. ${ }^{36,37}$ Thus, to increase the chances of capturing CTCs, a novel technology applying the combination of EpCAM and an EMT core transcription factor (Twist) was used to determine CTCs. Our results showed that the $\mathrm{Twist}^{+}$CTCs was significantly higher than $\mathrm{EpCAM}^{+}$CTCs in PoV. Further analysis demonstrated that the numbers of PoV CTCs and M-CTCs were positively correlated with lymph node metastasis, distant metastasis, and advanced TNM stage, instead of E-CTCs. Meanwhile, the number of CTCs and M-CTCs improved the diagnosis power of CA19-9 for distinguishing patients at stage I-II from those at stage III-IV, consistent with previous studies suggesting that M-CTCs have been linked to aggressive tumors. Collectively, the numbers of both portal CTCs and M-CTCs may act as potential biomarkers for tumor burdens for PC patients. ${ }^{22,23}$

Next, we validated CTCs and M-CTCs in PoV as prognostic markers in PC patients. Here, we used the Youden Index (= sensitivity + specificity-1) which could suggest the best cut-off value by fitting optimal sensitivity and specificity to determine the threshold in this work. We found that the baseline levels of $\geq 10 \mathrm{CTCs} / 5 \mathrm{~mL}$ or $\geq 8$ M-CTCs $/ 5 \mathrm{~mL}$ were associated with shorter OS. These results support the conclusion of previous studies that patients with a high number of CTCs in PoV may have an adverse prognosis and may require efficient managements to improve clinical outcomes. ${ }^{24,33,35}$

In conclusion, we provided a systematic approach in detecting PoV CTCs for PC diagnosis and prognosis prediction. Acquisition of the PoV samples in patients with PC via EUS-guided procedures has been proved to be safe and feasible. The number of PoV CTCs, especially M-CTCs, is positively correlated with the tumor burden, and has great potentials in distinguishing early and late PC patients, especially in combination with serum CA19-9. More importantly, PoV CTCs and M-CTCs can be used to predict prognosis in PC patients. Further study to investigate the clinical significance of PoV CTCs in a larger cohort of patients is needed in the near future.

\section{Data Sharing Statement}

We don't want to share individual deidentified participant data.

\section{Acknowledgment}

This work was supported by the National Natural Science Foundation of China (81802396), Natural Science
Foundation of Jiangsu Province (SBK2019022491 \& BK20180117), General Project of Nanjing Medical Science and Technology Development Project (YKK17077), Nanjing Science and Technology Development Plan Project (201715023), Nanjing Medical Science and Technology Development Key Project (ZKX18022), and Nanjing Science and technology project (201911038).

\section{Disclosure}

The authors report no conflicts of interest in this work.

\section{References}

1. Moore A, Donahue T. Pancreatic cancer. JAMA. 2019;322(14):1426. doi:10.1001/jama.2019.14699

2. Siegel RL, Miller KD, Jemal A. Cancer statistics, 2019. CA Cancer J Clin. 2019;69:7-34.

3. Rahib L, Smith BD, Aizenberg R, Rosenzweig AB, Fleshman JM, Matrisian LM. Projecting cancer incidence and deaths to 2030: the unexpected burden of thyroid, liver, and pancreas cancers in the United States. Cancer Res. 2014;74(11):2913-2921. doi:10.1158/ 0008-5472.CAN-14-0155

4. Mobarki M, Dumollard JM, Dal Col P, Camy F, Peoc'h M, Karpathiou G. Granular cell tumor a study of 42 cases and systemic review of the literature. Pathol Res Pract. 2020;216(4):152865. doi:10.1016/j.prp.2020.152865

5. Luo G, Liu C, Guo M, et al. Potential biomarkers in Lewis negative patients with pancreatic cancer. Ann Surg. 2017;265(4):800-805. doi:10.1097/SLA.0000000000001741

6. Zhou B, Xu JW, Cheng YG, et al. Early detection of pancreatic cancer: Where are we now and where are we going? Int J Cancer. 2017;141(2):231-241. doi:10.1002/ijc.30670

7. Pantel K, Speicher MR. The biology of circulating tumor cells. Oncogene. 2016;35(10):1216-1224. doi:10.1038/onc.2015.192

8. Miyamoto DT, Lee RJ, Kalinich M, et al. An RNA-based digital circulating tumor cell Signature is predictive of drug response and early dissemination in prostate cancer. Cancer Discov. 2018;8 (3):288-303. doi:10.1158/2159-8290.CD-16-1406

9. Guo W, Sun YF, Shen MN, et al. Circulating tumor cells with stem-like phenotypes for diagnosis, prognosis, and therapeutic response evaluation in hepatocellular carcinoma. Clin Cancer Res. 2018;24(9):2203-2213. doi:10.1158/1078-0432.CCR-17-1753

10. Deneve E, Riethdorf S, Ramos J, et al. Capture of viable circulating tumor cells in the liver of colorectal cancer patients. Clin Chem. 2013;59(9):1384-1392. doi:10.1373/clinchem.2013.202846

11. Nieto J, Grossbard ML, Kozuch P. Metastatic pancreatic cancer 2008: is the glass less empty? Oncologist. 2008;13(5):562-576. doi:10.1634/ theoncologist.2007-0181

12. Yu M, Stott S, Toner M, Maheswaran S, Haber DA. Circulating tumor cells: approaches to isolation and characterization. $J$ Cell Biol. 2011;192(3):373-382. doi:10.1083/jcb.201010021

13. Buscail E, Chiche L, Laurent C, et al. Tumor-proximal liquid biopsy to improve diagnostic and prognostic performances of circulating tumor cells. Mol Oncol. 2019;13(9):1811-1826. doi:10.1002/18780261.12534

14. Rahbari NN, Bork U, Kircher A, et al. Compartmental differences of circulating tumor cells in colorectal cancer. Ann Surg Oncol. 2012;19 (7):2195-2202. doi:10.1245/s10434-011-2178-1

15. Fang ZT, Zhang W, Wang GZ, et al. Circulating tumor cells in the central and peripheral venous compartment - assessing hematogenous dissemination after transarterial chemoembolization of hepatocellular carcinoma. Onco Targets Ther. 2014;7:1311-1318. doi:10.2147/OTT.S62605 
16. Reddy RM, Murlidhar V, Zhao L, et al. Pulmonary venous blood sampling significantly increases the yield of circulating tumor cells in early-stage lung cancer. J Thorac Cardiovasc Surg. 2016;151 (3):852-858. doi:10.1016/j.jtcvs.2015.09.126

17. Tien YW, Kuo HC, Ho BI, et al. A high circulating tumor cell count in portal vein predicts liver metastasis from periampullary or pancreatic cancer: a high portal venous CTC count predicts liver metastases. Medicine. 2016;95(16):e3407. doi:10.1097/MD.0000000000003407

18. Chapman CG, Waxman I. EUS-guided portal venous sampling of circulating tumor cells. Curr Gastroenterol Rep. 2019;21(12):68. doi:10.1007/s11894-019-0733-2

19. Catenacci DV, Chapman CG, Xu P, et al. Acquisition of portal venous circulating tumor cells from patients with pancreaticobiliary cancers by endoscopic ultrasound. Gastroenterology. 2015;149 (7):1794-1803 e1794. doi:10.1053/j.gastro.2015.08.050

20. Lee JS, Park SS, Lee YK, Norton JA, Jeffrey SS. Liquid biopsy in pancreatic ductal adenocarcinoma: current status of circulating tumor cells and circulating tumor DNA. Mol Oncol. 2019;13(8):1623-1650. doi:10.1002/1878-0261.12537

21. Konigsberg R, Obermayr E, Bises G, et al. Detection of EpCAM positive and negative circulating tumor cells in metastatic breast cancer patients. Acta Oncol. 2011;50(5):700-710. doi:10.3109/ 0284186X.2010.549151

22. Wei T, Zhang X, Zhang Q, et al. Vimentin-positive circulating tumor cells as a biomarker for diagnosis and treatment monitoring in patients with pancreatic cancer. Cancer Lett. 2019;452:237-243. doi:10.1016/j.canlet.2019.03.009

23. Zhao XH, Wang ZR, Chen CL, et al. Molecular detection of epithelial-mesenchymal transition markers in circulating tumor cells from pancreatic cancer patients: potential role in clinical practice. World $J$ Gastroenterol. 2019;25(1):138-150. doi:10.3748/wjg.v25.i1.138

24. Chapman CG, Ayoub F, Swei E, et al. Endoscopic ultrasound acquired portal venous circulating tumor cells predict progression free survival and overall survival in patients with pancreaticobiliary cancers. Pancreatology. 2020;20(8):1747-1754. doi:10.1016/j. pan.2020.10.039

25. Buscail L. Commentary: pancreatic cancer: is the worst to come? Int $J$ Epidemiol. 2017;46(6):1774-1775. doi:10.1093/ije/dyx143

26. Buscail E, Maulat C, Muscari F, Chiche L, Cordelier P, Dabernat S. Liquid biopsy approach for pancreatic ductal adenocarcinoma. Cancers. 2019;11(6):852. doi:10.3390/cancers 11060852

27. Chen J, Ye C, Dong J, et al. Metabolic classification of circulating tumor cells as a biomarker for metastasis and prognosis in breast cancer. J Transl Med. 2020;18(1):59. doi:10.1186/s12967-020-02 $237-8$
28. de Kruijff IE, Sieuwerts AM, Onstenk W, et al. Circulating tumor cell enumeration and characterization in metastatic castration-resistant prostate cancer patients treated with Cabazitaxel. Cancers. 2019;11 (8):1212. doi: $10.3390 /$ cancers 11081212

29. Cohen SJ, Punt CJ, Iannotti N, et al. Relationship of circulating tumor cells to tumor response, progression-free survival, and overall survival in patients with metastatic colorectal cancer. J Clin Oncol. 2008;26:3213-3221.

30. Gao Y, Zhu Y, Zhang Z, Zhang C, Huang X, Yuan Z. Clinical significance of pancreatic circulating tumor cells using combined negative enrichment and immunostaining-fluorescence in situ hybridization. J Exp Clin Cancer Res. 2016;35(1):66. doi:10.1186/ s13046-016-0340-0

31. Chapman CG, Waxman I. Portal-vein blood samples as a new diagnostic entity for pancreatic cancer. Expert Rev Gastroenterol Hepatol. 2016;10(6):665-667. doi:10.1080/17474124.2016.1176911

32. White MG, Lee A, Vicente D, et al. Measurement of portal vein blood circulating tumor cells is safe and may correlate with outcomes in resected pancreatic ductal adenocarcinoma. Ann Surg Oncol. 2021;28(8):4615-4622. doi:10.1245/s10434-020-09518-y

33. Dong X, Ma Y, Zhao X, et al. Spatial heterogeneity in epithelial to mesenchymal transition properties of circulating tumor cells associated with distant recurrence in pancreatic cancer patients. Ann Transl Med. 2020;8(11):676. doi:10.21037/atm-20-782

34. Gall TM, Jacob J, Frampton AE, et al. Reduced dissemination of circulating tumor cells with no-touch isolation surgical technique in patients with pancreatic cancer. JAMA Surg. 2014;149(5):482-485. doi:10.1001/jamasurg.2013.3643

35. Liu X, Li C, Li J, et al. Detection of CTCs in portal vein was associated with intrahepatic metastases and prognosis in patients with advanced pancreatic cancer. $J$ Cancer. 2018;9(11):2038-2045. doi:10.7150/jca.23989

36. Bidard FC, Huguet F, Louvet C, et al. Circulating tumor cells in locally advanced pancreatic adenocarcinoma: the ancillary CirCe 07 study to the LAP 07 trial. Ann Oncol. 2013;24(8):2057-2061. doi:10.1093/annonc/mdt176

37. Okubo K, Uenosono Y, Arigami T, et al. Clinical impact of circulating tumor cells and therapy response in pancreatic cancer. Eur J Surg Oncol. 2017;43:1050-1055.

\section{Publish your work in this journal}

Cancer Management and Research is an international, peer-reviewed open access journal focusing on cancer research and the optimal use of preventative and integrated treatment interventions to achieve improved outcomes, enhanced survival and quality of life for the cancer patient.
The manuscript management system is completely online and includes a very quick and fair peer-review system, which is all easy to use Visit http://www.dovepress.com/testimonials.php to read real quotes from published authors. 